\title{
The Chlamydia surveillance system in Sweden delivers relevant and accurate data: results from the system evaluation, 1997-2008
}

M Riera-Montes (Margarita.Riera@gmx.com) ${ }^{1,2}$, I Velicko ${ }^{1}$

1. Department of Epidemiology, Swedish Institute for Communicable Disease Control (SMI), Solna, Sweden

2. European Programme for Intervention Epidemiology Training (EPIET), European Centre for Disease Prevention and Control (ECDC), Stockholm, Sweden

Citation style for this article:

izzoli A, Hauffe HC, Carpi G, Vourc'h GI, Neteler M, Rosà R. Lyme borreliosis in Europe.

Euro Surveill. 2011;16(27):pii=19906. Available online: http://www.eurosurveillance.org/ViewArticle.aspx?Articleld=19906

This study evaluates the ability of the Chlamydia surveillance system to provide relevant information to inform prevention and control activities in Sweden. The system was evaluated, according to the Guidelines for Evaluating Public Health Surveillance Systems from the United States Centers for Disease Prevention and Control, using surveillance data from 1997 to 2008. We interviewed staff from the Swedish Institute for Communicable Disease Control, the National Board of Health and Welfare and one county medical officer (CMO). We conducted a survey among laboratories, CMOs and a sample of clinics. Satisfaction with the system was good for $86 \%$ of $\mathrm{CMO}$ s, all laboratories, and $99 \%$ of clinics. The interviewed stakeholders considered the system to deliver relevant and accurate information that is useful for health policy decision making. However, the objectives for Chlamydia surveillance should be clearly defined in order to adapt the system requirements, simplify data collection and improve timeliness.

\section{Introduction}

Chlamydia trachomatis $(\mathrm{Ct})$ is the most common bacterial sexually transmitted infection (STI) in Europe [1]. According to the latest report from the European Centre for Disease Prevention and Control (ECDC), 335,329 cases were notified in 2008 by 23 Member States of the European Union (EU), the European Economic Area and the European Free Trade Association (EEA/EFTA), with an incidence rate of 150 cases per 100,000. The incidence was even higher in the age group of 15-24 year-olds where it reached a rate of 976 cases per 100,000 . Underreporting is common and the real incidence in Europe is supposed to be much higher [1].

In Sweden, the absolute number as well as the incidence of Ct cases have been rising since 1997 (Figure 1), with a sharp increase in 2007 when diagnostic tests became available that detected the new $\mathrm{Ct}$ variant that had previously been missed with some of the standard tests [2]. The most affected group have been teenagers and young adults between 15 and 29 years of age, particularly women. This age group was responsible for most of the increase in incidence observed over the years, with little change over time in the other age groups. The $\mathrm{Ct}$ control strategy in Sweden includes free testing and treatment, active partner tracing and opportunistic screening, mainly of young women (when Chlamydia testing is offered during a visit to a gynaecologist or a youth health or STI clinic for other reasons (e.g. contraception counselling).

With the number of $\mathrm{Ct}$ cases steadily increasing, a reliable and functioning surveillance system is paramount to follow trends, inform public health action and monitor prevention and control interventions. Although the electronic surveillance system used in Sweden for all notifiable diseases (SmiNet) has been evaluated concerning the sensitivity and timeliness for four selected diseases in the past $[3,4]$, the Ct surveillance system has never been formally evaluated in Sweden. This study aimed to determine whether the current $\mathrm{Ct}$ surveillance system delivers relevant, accurate and timely information to those who need it in order to enable adequate prevention and control measures.

\section{FIGURE 1}

Chlamydia trachomatis infections notified per year and age group, Sweden 1997-2008*

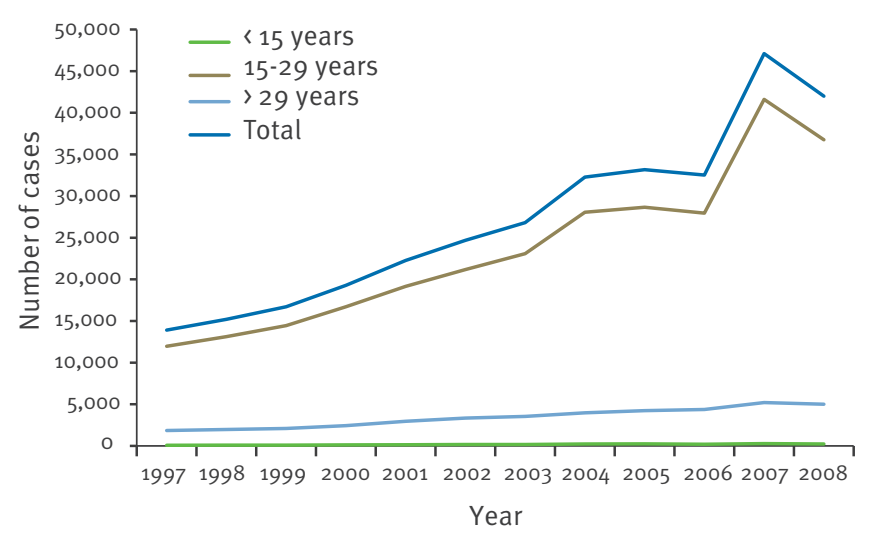




\section{Methods}

\section{Chlamydia surveillance in Sweden}

In Sweden, notification of $\mathrm{Ct}$ infection cases started on a voluntary basis for laboratories in 1982. Mandatory notification for clinicians was introduced in 1988 and for laboratories in 2004. Initially the reporting of cases was aggregated but case-based reporting was introduced in 1997 for clinical notifications and in 2005 for laboratory notifications. According to the case definition for Ct adopted in Sweden [5], all laboratoryconfirmed cases have to be reported to the national surveillance system SmiNet. Confirmation of the diagnosis is made by the 29 laboratories that test for $\mathrm{Ct}$.

The Ct surveillance system is comprehensive, including the entire Swedish population, and has two components: case-based reporting and aggregated reporting of the number of tests performed annually.

Case-based reporting is made through an electronic surveillance system (SmiNet) which was introduced in 1997 and upgraded and changed to web-based reporting in 2004 (SmiNet-2) [6]. Clinics and laboratories are required to report individual cases through SmiNet-2 using a webbased interface. Individual cases are reported anonymously using a modified code based on the unique personal identification number that all residents in Sweden have. The modified code itself is not unique, because reporting of STIs must by law be anonymous. Therefore laboratory and clinical notifications cannot be linked in the system. For the national surveillance, only clinical notifications are taken into account, since one case may have more than one laboratory report.

Aggregated reporting is a parallel voluntary surveillance system for laboratories to report the number of tests performed for $\mathrm{Ct}$ and the number of positives by sex and age group. This is reported electronically via SmiNet or on paper once a year. Although it is voluntary, all laboratories performing Chlamydia testing in Sweden report the data.

\section{Data flow}

Figure 2 shows the data flow between the different components of the $\mathrm{Ct}$ surveillance system, from the reporting sources to the data recipients. The data are collected at clinics and laboratories and entered in SmiNet through a web-based interface. The CMOs can access the data on cases reported in their own county, and SMI can access all the data. A summary of the data obtained from surveillance is published weekly on the SMI website, biannually in Epi-Aktuellt (SMl's official publication) and annually at SMI's Annual Epidemiological Report. This information is then used by SMI, CMOs and the National Board of Health and Welfare (SOS) to monitor trends and guide public health action concerning $\mathrm{Ct}$.

\section{Evaluation of performance attributes}

We followed the Guidelines for Evaluating Public Health Surveillance Systems from the United States Centers for Disease Prevention and Control (CDC) for the evaluation [7]. We aimed to evaluate the following system performance attributes: usefulness, simplicity, data quality, flexibility, acceptability, representativeness, timeliness and stability.

Usefulness, flexibility and acceptability were evaluated through semi-structured interviews conducted with key staff involved in Ct surveillance and control at the Swedish Institute for Communicable Disease Control (SMI) and the SoS as well as with the county medical officer from Västerbotten county. The CMO from Västerbotten county was chosen because of his expertise and research experience in Ct infections.

Data quality and timeliness were evaluated analysing the surveillance data for the period from 1997 to 2008 for clinical notifications and for the period from 2005 to 2008 for laboratory notifications.

We calculated the proportion of completeness for all variables, the proportion of invalid values and the median number of days with interquartile range (IQR) between the date of laboratory diagnosis and the date of notification. In order to assess timeliness we also calculated the percentage of notifications received within 24 hours, 7 days and 30 days of diagnosis (arbitrarily selected time spans) for the period from 2005 to 2008. We restricted the analysis to this period because the requirements were changed in 2004 to reporting within 24 hours of diagnosis.

\section{FIGURE 2}

Chlamydia surveillance data flow in Sweden

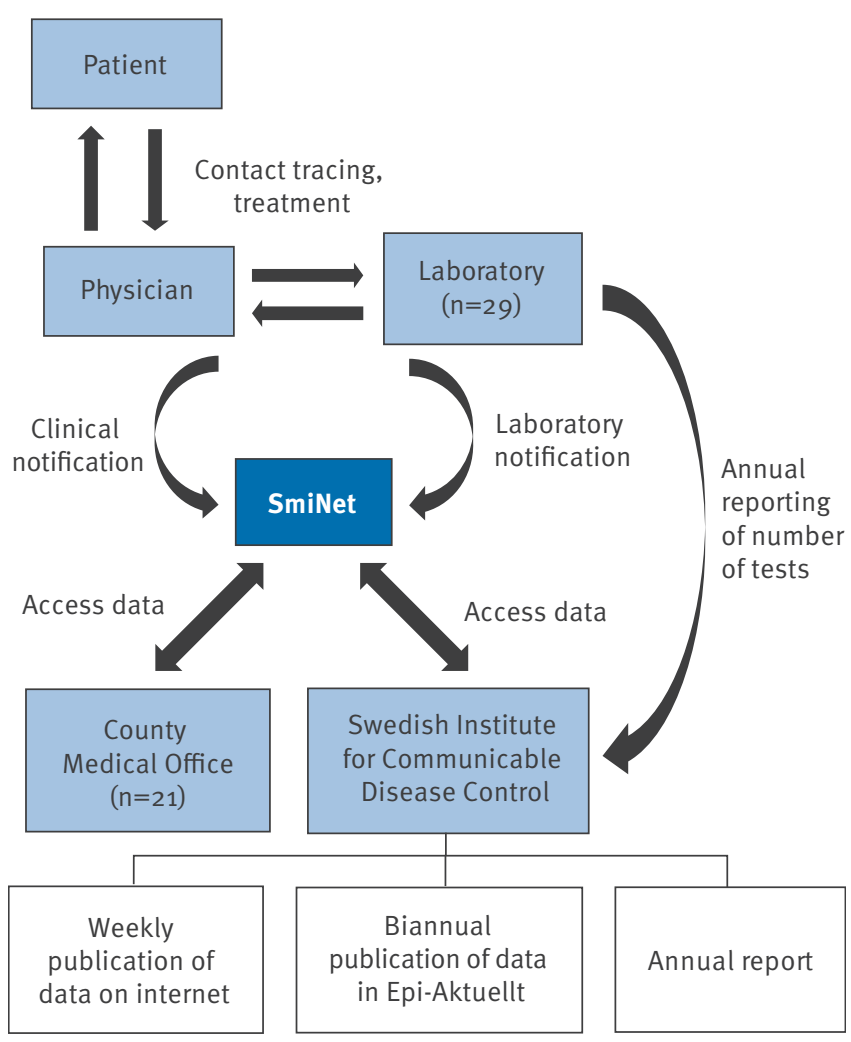


We considered that the most probable factor that could affect the system's representativeness in Sweden would be differences in access to healthcare between the different counties. The number of youth health clinics differs per county and some counties offer the possibility to request a free test through the Internet or even by mobile phone message. This can give different degrees of access to $\mathrm{Ct}$ testing in the different counties, and thus affect the representativeness of the $\mathrm{Ct}$ surveillance system. We calculated the number of youth clinics available per 10,000 population aged between 16 and 23 years in each county to get a rough estimate of access to $\mathrm{Ct}$ testing. The number of youth clinics per county was obtained from the official site for youth health clinics (www.umo.se). The population aged between 16 and 23 years per county was obtained from Statistics Sweden (www.scb.se) using the data for the year 2008. We chose this indicator because the majority of infections occur in the age group of 16-23 year-olds in Sweden and most of these infections are diagnosed and treated in youth clinics. We also examined differences between the sexes in the number of people screened per year for Ct infection to explore how this could affect the representativeness of the surveillance system.

Additionally, simplicity, data quality, acceptability, representativeness and stability were evaluated through self-administered questionnaires sent to all county medical offices (CMOs, $n=21$ ), all laboratories testing for $\mathrm{Ct}(\mathrm{n}=29)$ and a sample of clinics participating in $\mathrm{Ct}$ surveillance $(n=300)$. The questionnaires were sent by post and a reminder was sent by post or e-mail after four weeks to the CMOs and laboratories. No reminder was sent for the clinics due to logistics constraints. The sample of clinics was selected randomly among all clinics in Sweden that had reported at least 12 cases of $\mathrm{Ct}$ infection in 2008. We calculated that a sample of 200 clinics would be required to calculate proportions with a $95 \%$ confidence interval and a margin of error of less than 0.1. With an expected response rate of $70 \%$ based on previous surveys among healthcare workers in Sweden [8-10], we increased our sample by 30\% to compensate. The final sample was 300 clinics.

\section{Data analysis}

The responses from the CMOs and laboratories were entered manually in EpiData. The questionnaires from the clinics were scanned and exported to Excel. After data cleaning and validation, data analysis was carried out using Stata 10 (StataCorp) and MS Excel. Qualitative data obtained from the interviews and from open questions in the questionnaires were reviewed and organised into specific themes. These were then reviewed in relevance to the related attribute.

\section{Results}

We interviewed two staff from SMI, five staff from SoS and one county medical officer. All 21 CMOs, 26 of 29 laboratories (90\%) and 183 of 300 clinics (61\%) returned the questionnaire.

\section{Purpose of the surveillance system}

The overall aim of the $\mathrm{Ct}$ surveillance system as part of the national surveillance system is to meet the requirements of the Swedish Communicable Disease Act [11] in order to protect the population against the spread of infectious diseases. However, we could not identify any documents stating the specific objectives for $\mathrm{Ct}$ surveillance. That was a justification to use CDC's guidelines for the evaluation of the surveillance system and not to evaluate the system against its objectives. According to the staff involved in Chlamydia surveillance the objectives were: to estimate the incidence of Chlamydia infection in Sweden, to monitor trends in notified cases by age, sex and reporting county, and to identify potential risk groups for further preventive interventions.

\section{Usefulness}

All the stakeholders interviewed agreed that the data collected through surveillance were useful for health policy decision making, stimulating research and monitoring interventions aimed at controlling and preventing $\mathrm{Ct}$ infection. As a direct result of the analysis and interpretation of surveillance data showing a steady increase in the number of cases in recent years, a new Chlamydia National Action Plan was launched in 2009 [12]. This plan aims at reducing prevalence of $\mathrm{Ct}$ infection by 2014 through increased use of condoms among teenagers and young adults (15-29 years-old), increased awareness and understanding of $\mathrm{Ct}$ infection in the population, increased access to testing, counselling and treatment and improvement in sexual education in schools [12]. In addition, there is a KAP (Knowledge, Attitudes and Practices) study underway among youths in Sweden (UngKAB, Socialstyrelsen) to improve understanding of underlying factors influencing the increase in the number of cases and to identify opportunities for prevention and control.

\section{Simplicity}

Data collection

The data collected for surveillance has a set of compulsory fields and a set of voluntary fields. For the clinical notifications, the following fields are compulsory: registration date (assigned automatically by SmiNet), diagnosis (disease), type of patient identification (ID) used, patient ID, age, sex, county of residence, type of clinic, reporting clinic and responsible physician's name. The median time needed to collect this information in the studied period was 20 minutes (range: 1-180 minutes). The information needed for the laboratory notification is obtained from the laboratory request form. The following fields are compulsory: registration date (assigned automatically by SmiNet), diagnosis (disease), laboratory, type of report, type of patient ID used, patient ID, age, responsible laboratory physician, referring clinic and county of the clinic.

Data management

Once the information is collected in the clinics, the data must be manually entered into SmiNet (or sent 
in paper to the CMO). Fifty-three percent of the clinics (96 of 182) reported entering data less than once a week, while only $6 \%$ (11 of 182 ) did so daily. The median time to fill in the form was 5 minutes (range: 1-30 minutes).

Laboratory notifications can be done automatically by direct link of the laboratory database to SmiNet or manually entering the data into SmiNet. Fifteen of 24 laboratories reported having automatic notification. The data was entered daily in all of the laboratories with automatic reporting and in seven of the nine laboratories with manual reporting.

After the notifications are entered into SmiNet, they must be approved by the CMOs. This was done daily by 18 of the 21 CMOs. Twelve CMOs reported checking for double reporting using the laboratory number, the numeric code or the date of sampling.

Ninety-six percent of the clinics (173 of 180) and all laboratories reported dedicating less than 5 hours per week to activities related to surveillance, while 15 of 21 CMOs reported less than 10 hours.

At SMI, the notifications are reviewed weekly by an epidemiologist. Regular contact is maintained with CMOs when required.

\section{Flexibility}

SmiNet was introduced in 1997 and upgraded to SmiNet-2 in 2004. Before 2004 all the data was sent to the CMOs or SMI to be entered, but since the introduction of SmiNet-2 notifications have been entered directly by the clinics and laboratories. The notification reports in SmiNet for Ct can be easily modified if new variables need to be added or unnecessary ones eliminated. However, any changes must be approved by the CMOs, SMI and SoS.

\section{Data quality}

Perceived data quality

Fifty-seven percent of the clinics (102 of 180) reported never or only occasionally filling in all the information requested in the notification form. The main reason given for this was that the requested information was considered to be irrelevant for Ct surveillance.

Observed completeness of the data

In the period under evaluation there were 325,925 clinical notifications and 104,642 laboratory notifications. The compulsory variables were $100 \%$ complete for all the years for both types of notifications. Tables 1 and 2 show the percentages of completeness for selected non-compulsory variables in clinical and laboratory notifications for the time period under evaluation. We also examined the percentage of clinical notifications with invalid values. For most variables, it was under $1 \%$. However, for infection date and onset date, this percentage was as high as $7 \%$.

\section{Acceptability}

Eighteen of the 21 CMOs, all the laboratories and 168 of 169 of the clinics (99\%) reported being satisfied or

\section{TABLE 2}

Completeness of non-compulsory variables (\%) in laboratory notifications for Chlamydia trachomatis infection, Sweden 2005-2008

\begin{tabular}{|l|c|c|c|c|}
\hline Variable & 2005 & 2006 & 2007 & 2008 \\
\hline Sex & 99 & 100 & 100 & 100 \\
\hline Date of receipt of sample & 79 & 80 & 87 & 86 \\
\hline Date of testing & 99 & 99 & 98 & 97 \\
\hline Sample material & 98 & 94 & 98 & 98 \\
\hline Test number & 86 & 96 & 97 & 97 \\
\hline
\end{tabular}

\section{TABLE 1}

Completeness of non-compulsory variables (\%) in clinical notifications for Chlamydia trachomatis infection, Sweden 1997-2008

\begin{tabular}{|l|c|c|c|c|c|c|c|c|c|c|c|c|}
\hline Variable & 1997 & 1998 & 1999 & 2000 & 2001 & 2002 & 2003 & 2004 & 2005 & 2006 & 2007 & 2008 \\
\hline Date of diagnosis & 4 & 60 & 85 & 89 & 89 & 92 & 93 & 92 & 95 & 94 & 93 & 92 \\
\hline Type of infection & 5 & 77 & 78 & 90 & 93 & 97 & 97 & 96 & 92 & 91 & 91 & 90 \\
\hline Reason for examination & 4 & 43 & 56 & 56 & 59 & 62 & 62 & 64 & 70 & 81 & 87 & 87 \\
\hline Infection date & 1 & 9 & 18 & 20 & 28 & 27 & 27 & 32 & 45 & 38 & 37 & 29 \\
\hline Onset date & 1 & 7 & 11 & 12 & 13 & 14 & 14 & 13 & 15 & 18 & 17 & 16 \\
\hline Country of infection & 9 & 47 & 67 & 79 & 85 & 89 & 90 & 92 & 93 & 92 & 91 & 91 \\
\hline Place of infection & 5 & 37 & 55 & 54 & 56 & 62 & 64 & 66 & 66 & 64 & 60 & 58 \\
\hline Place of onset & 1 & 11 & 16 & 17 & 19 & 21 & 23 & 25 & 32 & 33 & 32 & 33 \\
\hline Route of transmission & 8 & 71 & 92 & 94 & 94 & 96 & 98 & 98 & 96 & 95 & 95 & 89 \\
\hline Country of birth & 0 & 0 & 0 & 0 & 0 & 0 & 0 & 0 & 6 & 23 & 29 & 34 \\
\hline Reporting laboratory & NA & NA & NA & NA & NA & NA & NA & 1 & 31 & 60 & 76 & 77 \\
\hline Laboratory number & NA & NA & NA & NA & NA & NA & NA & 1 & 18 & 59 & 77 & 76 \\
\hline
\end{tabular}


very satisfied with the $\mathrm{Ct}$ surveillance system. CMOs and laboratories mentioned as the main disadvantage of the system the impossibility to use the personal identification number in the reporting, while clinics reported the lack of access to the report once is sent as the main disadvantage.

\section{Representativeness}

Perceived representativeness

On the questionnaires, 12 CMOs reported having an estimation of the degree of underreporting in their counties. They estimated it to be between o and $10 \%$ with a median of $4 \%$. In the interviews, the stakeholders estimated that they had $5 \%$ underreporting based on the difference in the number of clinical and laboratory notifications.

\section{FIGURE 3}

Median delay time (in days) between date of diagnosis and date of laboratory notification of Chlamydia trachomatis infections, Sweden 2005-2008 $(n=104,642)$

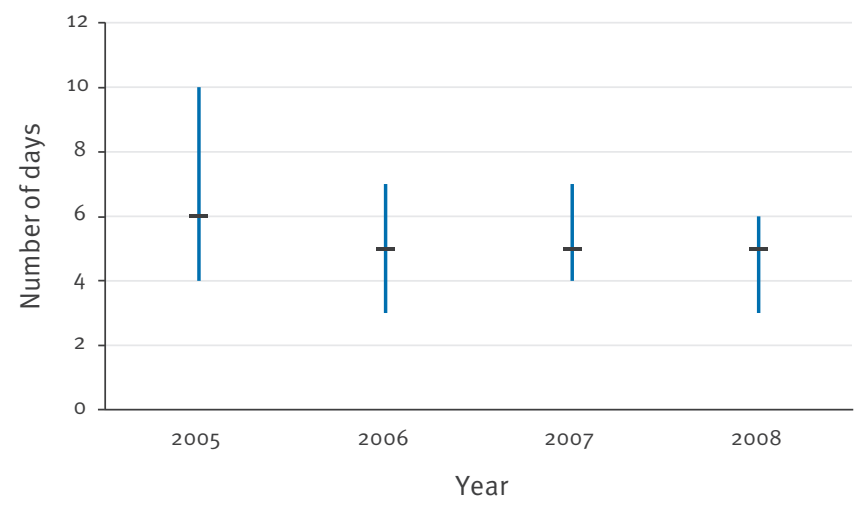

Observed representativeness

In Sweden, there are between one and 10 youth clinics per 10,000 population aged between 16 and 23 years per county, with a median of three. There was no correlation between number of youth clinics per 10,000 16-23 year-olds and Chlamydia incidence in the same age group (Spearman's rank correlation test, $p=0.8812$ ).

We also examined differences between sexes in the number of tests performed annually for Ct infection for the period for which such information was available. Between the years 2000 and 2008 almost 3 million tests were performed among women compared with just under 1 million among men. Although the number of tests increased over the years, especially among men, there were still 2.6 times more tests per year among women than among men in 2008. However, the rate of positive tests was higher among men.

\section{Timeliness}

\section{Perceived timeliness}

In the interviews, publication of the data on the SMI website once a week was considered to be timely enough. However, publication only every six months of the aggregated reporting of the number of tests was considered to be insufficient.

Observed timeliness

The median delay between the date of the laboratory test and the date of notification to SmiNet was 5 days (interquartile range (IQR): IQR 3-7 days) for laboratory notifications and 11 days (IQR 6-20 days) for clinical notifications for the whole study period (Figures 3 and 4).

Overall, $1 \%$ of all laboratory notifications during the study period were notified within 24 hours, $85 \%$ within

\section{FIGURE 4}

Median delay time (in days) between the date of diagnosis and the date of clinical notifications of Chlamydia trachomatis infections, Sweden 1997-2008 $(n=325,925)$

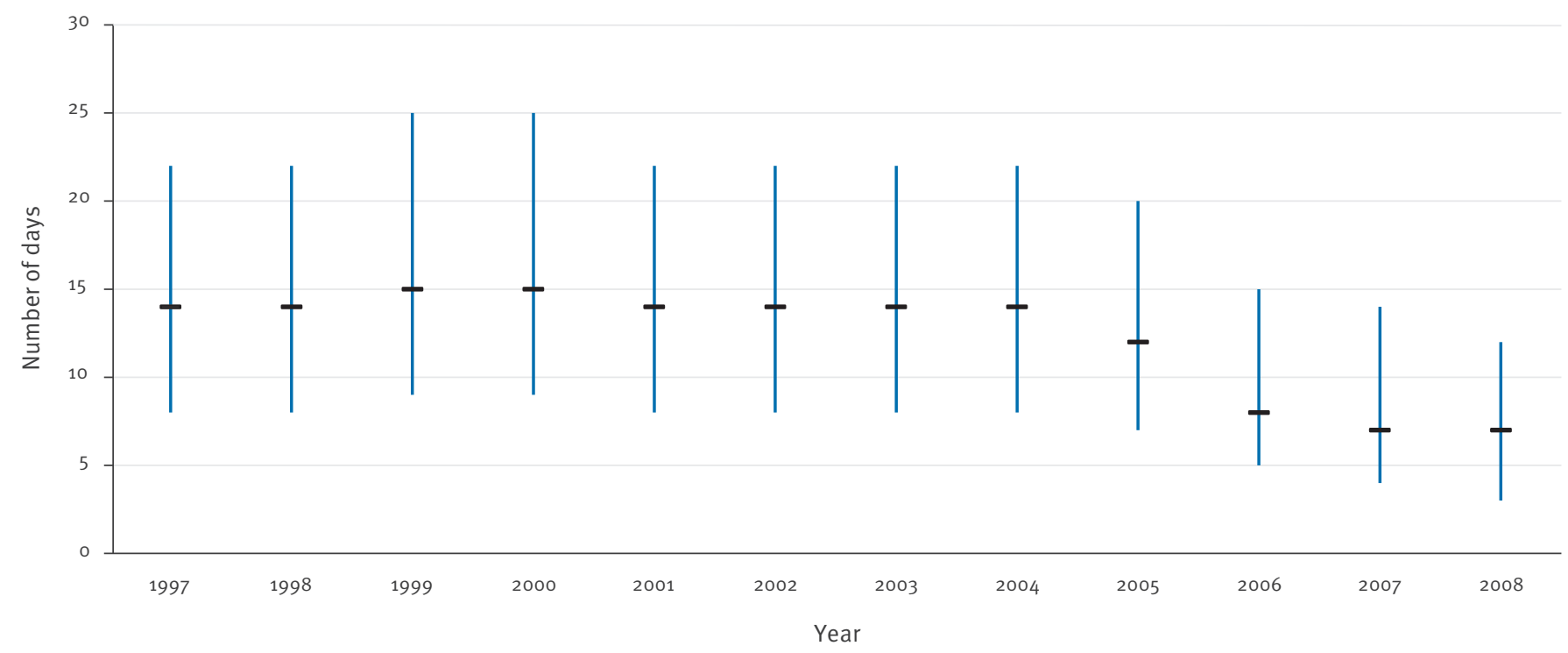


7 days and $99 \%$ within 30 days. For clinical notifications the figures were $9 \%, 45 \%$ and $91 \%$, respectively.

\section{Stability}

Five per cent of the clinics, $10 \%$ of the laboratories and $81 \%$ of the CMOs reported having technical problems with SmiNet. The frequency of these problems was once a year or less for $97 \%$ of the clinics and $75 \%$ of the laboratories, and at least once a month for $88 \%$ of CMOs. The problems most frequently reported were system freeze and problems with login. When asked about the use of SmiNet technical support, $54 \%$ of the clinics, $58 \%$ of the laboratories and all the CMOs reported always receiving it when required.

\section{Discussion}

We aimed to evaluate the $\mathrm{Ct}$ surveillance system in Sweden by describing the system and measuring the usefulness, simplicity, data quality, acceptability, representativeness, timeliness and stability of the system. We could not identify any documents stating the specific objectives for the Ct surveillance system. Defining the objectives of the system would be crucial to establish whether the current system is adequate for $\mathrm{Ct}$ surveillance and better define the improvements necessary for the system to deliver the relevant information needed for action.

An important challenge with the surveillance system in Sweden is the anonymous notification of cases which makes automatic linkage between clinical and laboratory notifications impossible. Due to this complexity of linkage it was decided to consider only the clinical notifications with epidemiological information for case counting. The laboratory notifications, mandatory according to the Communicable Disease Act, are therefore not used for routine surveillance (but for could be used for the research). This means that we may miss laboratory-confirmed cases for which a clinical notification does not exist. This lack of unique personal identification number (which would allow linking clinical and laboratory notifications) was perceived as an important disadvantage of the $\mathrm{Ct}$ surveillance system by all stakeholders.

The simplicity of the Ct surveillance system was perceived as adequate by the respondents. System users considered the data flow as good (fast enough and technically less problematic than manual reporting) between the different levels (clinics/laboratories, CMOs and SMI). The number of compulsory variables needed to fill in a clinical or laboratory notification is low. However, the total number of variables in the clinical notification is high. The variation between clinics in the time needed to collect all the information for the clinical notification may be related to how thorough they are in their data collection, although we did not assess this aspect. For the laboratories, the introduction of automatic reporting linked to the databases has made the reporting easier and less resourceintensive. Simplicity of use and speed were the main advantages of the system perceived by CMOs, clinics and laboratories.

The information gathered through surveillance was considered to be useful by health policy decision makers. All interviewed stakeholders reported using the information gathered through surveillance to decide on public health action, such as the implementation of a new Chlamydia National Action Plan in 2009 [12]. However, there were suggestions to collect more information on the social background and sexual behaviour of cases. This would help to identify particular risk groups and to better target prevention activities but would be time- and resource-consuming. Finland has a sentinel surveillance network for STIs where detailed information concerning risk behaviour is collected through a self-administered anonymous questionnaire in 12 clinics around the country [13]. A similar approach could be explored in Sweden.

The $\mathrm{Ct}$ surveillance system is flexible and modifying the notification forms is possible and relatively easy to do. However, agreement between all stakeholders (SMI, SoS and (MOs) is needed before any changes can be implemented, which can result in delays. Data quality was considered to be 'good' for compulsory variables and for voluntary variables perceived as important for Ct surveillance. Variables like infection date, onset date, place of onset, place of infection and country of birth had a low completeness rate. They were considered to be 'irrelevant' and it was suggested to remove them from the notification because this information is rarely available.

The general acceptability of the Ct surveillance system is high. Most CMOs, laboratories and clinics are satisfied or very satisfied with the system.

Representativeness was very difficult to evaluate. $\mathrm{Ct}$ surveillance comprises all laboratory-confirmed cases in Sweden. However, there is no information on the real prevalence of the infection among the Swedish population, so it is very difficult to evaluate the number of unreported cases or the number of undiagnosed cases. The interviewed CMOs and the SMI estimated underreporting to be around $5 \%$ based on the discrepancy between the number of laboratory and clinical notifications. All stakeholders interviewed agreed that the real incidence was probably higher than reported although there are no prevalence studies for $\mathrm{Ct}$ in Sweden to compare it with. According to the stakeholders, the trends obtained through the surveillance system should be considered as accurate. Although there is no data on $\mathrm{Ct}$ prevalence in Sweden and Ct incidence is underreported (due to many asymptomatic and untested persons), general trends are considered to mirror reality quite well.

The number of tests performed among women was higher than among men in all studied years since 2000. However, the positivity rate was higher among 
men. Rather than a shortcoming of the surveillance system itself, this could indicate differences in health seeking behaviour among men, or that prevention activities like opportunistic screening are more targeted at women. We believe that surveillance system is mirroring the real distribution of Chlamydia cases in the population by age group, with underrepresentation of cases among men due to the reasons mentioned above.

The timeliness of case reporting and data publication was adequate, and it improved during the period evaluated. However, only $1 \%$ of laboratory notifications and $9 \%$ of clinical notifications were notified within 24 hours of diagnosis as required by the Communicable Diseases Act. The weekly updates of $\mathrm{Ct}$ surveillance data on the SMI website was considered timely enough by all stakeholders. From a surveillance point of view, the notification period could be extended to one week to match the frequency of reporting. This could be improved by implementing automated data reporting from the laboratories, similar to what is already done with the case-based reporting. However, this would require the laboratories to invest in the development of programmes for automated data transfer, which can take time to implement.

The system stability was perceived as 'good' with few technical problems reported and efficient technical support when required.

In conclusion, the $\mathrm{Ct}$ surveillance system in Sweden delivers relevant and accurate data to inform public health action. However, the system could be improved further by implementing the following recommendations:

- Establish clear objectives for $\mathrm{Ct}$ surveillance in order to adapt the current system to the needs of stakeholders;

- Equate $\mathrm{Ct}$ with other notifiable diseases enabling reporting with the personal identification number to avoid case duplication and evaluation of reinfections;

- Adapt the clinical and laboratory notification forms specifically for $\mathrm{Ct}$ eliminating unnecessary variables in order to simplify data collection and improve data quality, acceptability and timeliness;

- Extend automatic reporting to all laboratories to increase timeliness.

\section{Acknowledgments}

We would like to thank Malin Arneborn (SMI) and Jens Boman (Skåne region medical officer) for their contribution to the evaluation design and for the critical review of the manuscript.

*Erratum: In the version originally published on 7 July 2011, the curves in Figure 1 were assigned to the wrong age groups. This mistake was corrected on 14 July 2011.
References

1. European Centre for Disease Prevention and Control (ECDC). Annual Epidemiological Report on Communicable Diseases in Europe 2010. Stockholm: ECDC; 2010. Available from: http://ecdc.europa.eu/en/publications/Publications/1011 SUR_Annual_Epidemiological_Report_on_Communicable_ Diseases_in_Europe.pdf

2. Velicko I, Kühlmann-Berenzon S, Blaxhult A. Reasons for the sharp increase of genital chlamydia infections reported in the first months of 2007 in Sweden. Euro Surveill. 2007:12 (10):pii=737. Available from: http://www.eurosurveillance.org/ ViewArticle.aspx?Articleld $=737$

3. Jansson A, Arneborn M, Skärlund K, Ekdahl K. Timeliness of case reporting in the Swedish statutory surveillance of communicable diseases 1998--2002. Scand J Infect Dis. 2004;36(11-12):865-72.

4. Jansson A, Arneborn M, Ekdahl K. Sensitivity of the Swedish statutory surveillance system for communicable diseases 1998-2002, assessed by the capture-recapture method. Epidemiol Infect. 2005;133(3):401-7.

5. Falldefinitioner vid anmälan enligt smittskyddslagen. [Case definitions - for notification according to the Communicable Diseases Act]. Stockholm, Socialstyrelsen: 2008. Swedish. Available from: http://www.socialstyrelsen.se/ publikationer2008/2008-130-11

6. Rolfhamre P, Jansson A, Arneborn M, Ekdahl K. SmiNet-2: Description of an internet-based surveillance system for communicable diseases in Sweden. Euro Surveill. 2006;11(5):pii=626. Available from: http://www. eurosurveillance.org/ViewArticle.aspx?Articleld $=626$

7. German RR, Lee LM, Horan JM, Milstein RL, Pertowski CA, Waller MN et al. Updated guidelines for evaluating public health surveillance systems: recommendations from the Guidelines Working Group. MMWR Recomm Rep. 2001;50(RR13):1-35; quiz CE31-37.

8. Aneblom G, Lundborg CS, Carlsten A, Eurenius K, Tydén T. Emergency contraceptive pills over-the-counter: practices and attitudes of pharmacy and nurse-midwife providers. Patient Educ Couns. 2004:55(1):129-35.

9. Löfgren A, Hagberg J, Arrelöv B, Ponzer S, Alexanderson K. Frequency and nature of problems associated with sickness certification tasks: a cross-sectional questionnaire study of 5455 physicians. Scand J Prim Health. 2007;25(3):178-85.

10. Swartling MS, Hagberg J, Alexanderson K, Wahlström RA. Sick-listing as a psychosocial work problem: a survey of 3997 Swedish physicians. J Occup Rehabil. 2007;17(3):398-408.

11. Smittskyddslag 2004:168. [Communicable Disease Act 2004:168]. Stockholm: Socialdepartementet; 7 Apr 2004. Swedish. Available from: http://www.notisum.se/rnp/sls/ sfs/20040168.pdf

12. Nationell handlingsplan för klamydiaprevention - med fokus på ungdomar och unga vuxna 2009-2014. [National action plan for chlamydia prevention - focusing on adolescents and young adults 2009-2014]. Stockholm: Socialstyrelsen:.2009. Swedish. Available from: http://www.socialstyrelsen.se/ publikationer2009/2009-126-180

13. Hiltunen-Back E, Haikala O, Kautiainen H, Ruutu P, Paavonen J Reunala T. Nationwide increase of Chlamydia trachomatis infection in Finland: highest rise among adolescent women and men. Sex Transm Dis. 2003;30(10):737-41. 